\title{
Endosalpingiosis: A Case Report
}

\author{
Eddy Hartono ${ }^{1}$,, Nusratuddin Abdullah ${ }^{1}$, Rahmawati ${ }^{1}$, Ni Ketut Sungowati ${ }^{2}$ \\ ${ }^{1}$ Division of Fertility and Reproductive Endocrinology, Department of Obstetrics and Gynecology, Faculty of Medicine, Hasanuddin \\ University, Makassar, Indonesia \\ ${ }^{2}$ Department of Anatomic Pathology, Faculty of Medicine, Hasanuddin University, Makassar, Indonesia
}

\section{Email address:}

eddyhartonospog@gmail.com (E. Hartono)

${ }^{*}$ Corresponding author

\section{To cite this article:}

Eddy Hartono, Nusratuddin Abdullah, Rahmawati, Ni Ketut Sungowati. Endosalpingiosis: A Case Report. Journal of Gynecology and Obstetrics. Vol. 4, No. 5, 2016, pp. 30-33. doi: 10.11648/j.jgo.20160405.12

Received: August 30, 2016; Accepted: September 8, 2016; Published: September 28, 2016

\begin{abstract}
Endosalpingiosis is a condition in which fallopian tube-like epithelium is found outside the fallopian tube. It is a non-neoplastic lesion of Mullerian system. This disease was first discovered by Sampson in 1930. Endosalpingiosis incidence is rare compared to endometriosis (about 5-10\%) and it is commonly found in reproductive-age women. Endosalpingiosis diagnosis is confirmed by histopathological examination. The objective of this case report is to increase our knowledge about endosalpingiosis. We reported a female, 36 years old, P1A0, with lower abdominal pain for the last 4 years. The woman had a regular menstruation every month with dysmenorrhea. On physical examination, it obtained normal vital signs and normal nutritional status with BMI of 23.29. On gynecological examination, it showed $7 \times 6 \mathrm{~cm}$ palpable cystic mass in the right adnexa and mobile. The result of transvaginal ultrasound showed the presence of hypoechoic masses on the right and left adnexa with $7.97 \times 7.95 \mathrm{~cm}$ and $3.28 \times 2.87 \mathrm{~cm}$ in size. During laparoscopy, right paratubal cyst and pseudocyst on the left adnexa appeared with $8 \times 8 \mathrm{~cm}$ and $5 \times 4 \mathrm{~cm}$ in size, yellowish-white color, and uterus to rectum adhesion. Adhesiolysis as well as bilateral cystectomy were conducted and then the histopathological result showed paratubal serous cystadenoma with endosalpingiosis. Endosalpingiosis is a rare case. The attention to this rare case will prevent us from making a wrong diagnosis and overtreatment.
\end{abstract}

Keywords: Endosalpingiosis, Histopathology, Mullerian System

\section{Introduction}

Endosalpingiosis is a non-neoplastic lesion of Mullerian system, which is rarely found. Endosalpingiosis is a condition where the epithelium cell of the fallopian tubes found outside of the fallopian tube, the cells can be found in the peritoneum, tissue of subperitoneal, omentum, retroperitoneal, urinary vesicles, can even be found in other organs outside the pelvic as in the thoracic region, duct choledochal and in the axillary lymph nodes. [1,2] This disease was first disclosed by Sampson in 1930. [3, 4] Some scientists have also been reported cases of such endosalpingiosis such as Rose Bermejo et al in 2012 and Mohiedean Ghofrani MD in 2011.[1,5] The incidence of endosalpingiosis is a little compared with the incidence of endometriosis, which is about $5-10 \%$ and is commonly found in women of reproductive age aged between 30-50 years than in women of postmenopausal age.[6] Our objective of this case report is to increase our knowledge about endosalpingiosis
Endosalpingiosis exact cause remains unclear, but several theories have been raised by scientists among others implantation theory by Clement and Young who reported 9 cases of Mullerianosis that occurs in patients with a history of pelvic area surgery. $[6,7]$ Other allegations, the presence of chronic salpingitis may precipitate tubal epithelium cell implantation in other places as well as the emergence of serous ovarian tumors can trigger the maturation of tumor implantation.[8] The presence of endosalpingiosis found outside pelvic area also indicates that the displacement torn of epithelium cells through the flow of lymph or blood vessels as a mechanism that may occur. $[3,6]$

Endosalpingiosis often discovered by chance along with ovarian tumors, endometriosis, and uterine tumors. Research conducted by L Prentice et al in 2012 revealed that about $34.5 \%$ of endosalpingiosis found along with endometriosis and $40 \%$ of endosalpingiosis constitutes postmenopausal woman. [9] 
In endosalpingiosis there is not found cytogenetic endometrial such as stroma responded to hormonal cycle (menstruation) so as to be distinguished with endometriosis, and does not indicate a stratification of cells and atypical mitotic activity or that must be differentiated with carcinoma. [1]

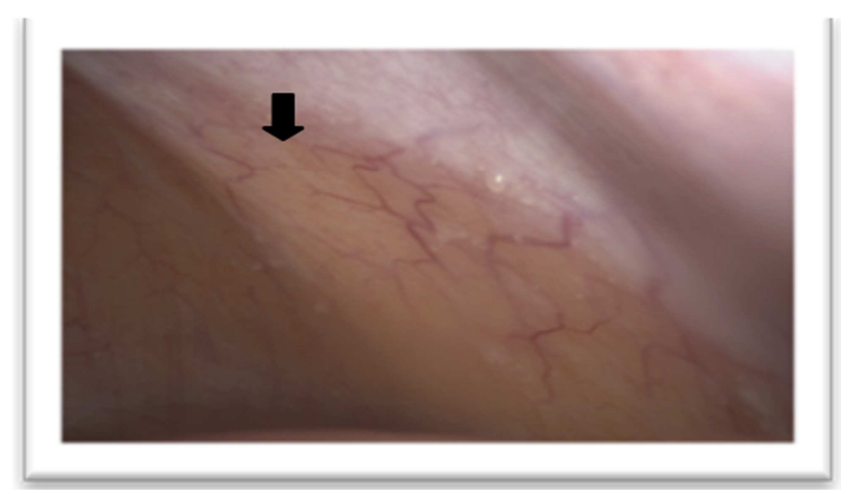

Figure 1. Overview of microscopic Endosalpingiosis (quoted from literature 10).

Endosalpingiosis diagnosis is made based on the results of microscopic examination by a pathologist through an incision (e.g., biopsy). Macroscopically, though rarely evident, it can appear as multiple small cysts colored in yellowish white (1$2 \mathrm{~mm}$ ) as shown in Figure 1 and more rarely as large cysts that can be confused as neoplasms. $[1,5]$

Microscopically it appears glands and tubules limited by the type of fallopian tube cells (i.e., cells epithelium ciliated) surrounded by fibrous stroma, sometimes also surrounded by chronic inflammatory infiltrate. Usually also looks calcification or bodies' psammoma. [5, 11] Another case if a cyst with tubal epithelium cells are surrounded by a type of endometrial stroma, that certain condition is not endosalpingiosis but rather a variation of endometriosis. Endosalpingiosis sometimes also be found in the lymph nodes that could be interpreted as metastatic adenocarcinoma. [11]

Here is an overview of endosalpingiosis under a microscope with high magnification, seemed typical of cysts bounded by epithelium cell ciliated after staining $\mathrm{H} \& \mathrm{E}$. [11]
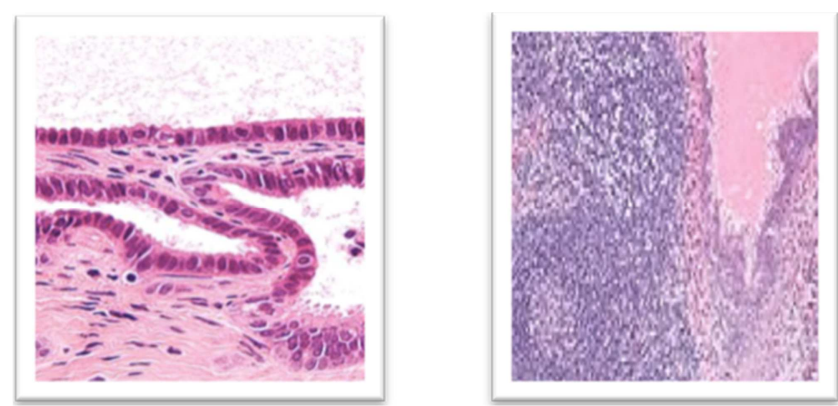

Figure 2. Appears a cyst with a gap bounded by ciliated epithelial cells (left) and can also be found in the lymph nodes (right) (quoted from the literature 11).

\section{Case}

A woman, 36 years old, P1A0, came to the clinic with complaints of lower abdominal pain since the last 4 years.
She has regular menstruation each month with 4-5 days menstruation with dysmenorrhea. History of vaginal discharge and fever denied. She also has history of vaginal delivery in 2004 and never used contraceptives. In the physical examination we obtained vital signs within normal limits, normal nutritional status with IMT of 23.29. From gynecological examination we found palpable cystic mass in the right adnexal size $7 \times 6 \mathrm{~cm}$, mobile, noticeably tenderness. The results of trans-vaginal USG gynecology appears the presence of adnexal masses hypoechoic on the right and left of each measure $7.97 \times 3.28 \times 7.95 \mathrm{~cm}$ and $2.87 \mathrm{~cm}$ (figure 3).
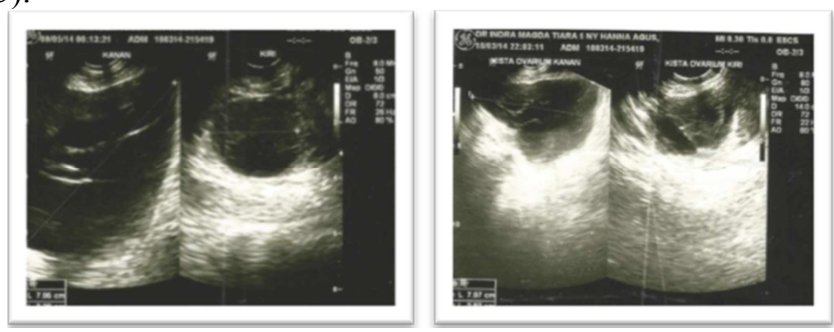

Figure 3. Overview of gynecology transabdominal USG, hypoechoic mass appears on the right and left adnexal.

Via laparoscopy, appears uterine size $7 \times 5 \mathrm{~cm}$ with slippery surfaces experiencing adhesions with rectum in the posteriorly (Figure 4a), conducted adhesiolysis. It also seems right paratubal cyst measuring about $8 \times 8 \mathrm{~cm}$ (Figure $4 \mathrm{~b}$ ) and pseudocyst on the left adnexal size of $5 \times 4 \mathrm{~cm}$ (Figure 4c), smooth, fluid-filled yellowish white. Cystectomy performed on both the cyst. Once bleeding is controlled, tubal patency test is done and both tubes patents. Both cysts are examined in anatomic pathology laboratory of Wahidin Sudirohusodo Hospital with histopathological results of cystadenoma serosum and endosalpingiosis. Postoperative day 10 the patient came for a control with both surgical wound and complaints of abdominal pain that decreases.
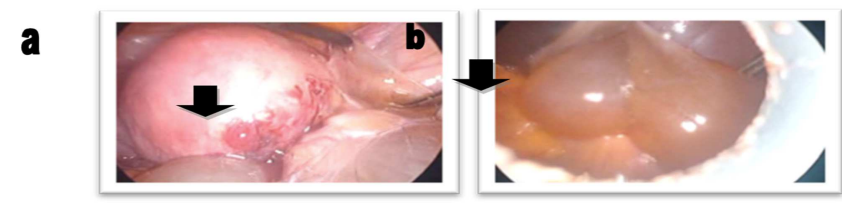

C

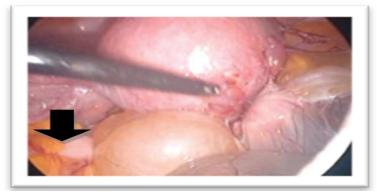

Figure 4. Uterine (a), cyst right paratubal (b) and left pseudocyst (c) appeared before laparoscopy.

Here is an overview of histopathology of the tissue of cyst removed. In Figure 5 below the tissue shows the cyst which consists of connective tissue covered with cuboidal epithelial layer, on the other part there is a connective tissue structure covered with 1-2 layers of ciliated columnar epithelium, the core is not atypical, thus diagnosed as Cystadenoma serosum paratubal with endosalpingiosis. 

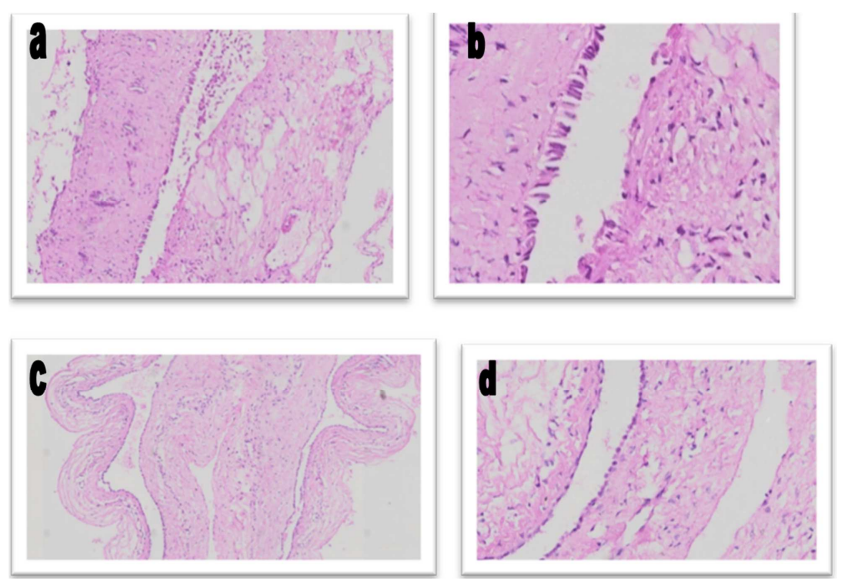

Figure 5. Histopathologic features of paratubal cyst, appears connective tissue covered with ciliated columnar epithelium ( $a$ and $b$ ) and connective tissue covered with cuboidal layer (c and d).

\section{Discussion}

Endosalpingiosis is a rare case found and the exact cause is still unknown. Clinically and experimentally revealed two mechanisms of pathogenesis of endosalpingiosis, namely metastatic spread from epithelium cells to the area and the presence of ectopic and metaplasia in the mesothelium pelvic, where the mesothelium pelvic and lower abdominal is ontogenetic with Mullerian duct known as the initial formation of the female genital tract. However, most scientists adhere that pathogenesis of endosalpingiosis is due to the change of metaplastic from pluripotent peritoneum. In addition the migration of Mullerian tissues during embryonic development or during surgical intervention is another mechanism that might occur. $[1,3,6]$

Symptoms of this disease is not typical, some scientists discovered various symptoms and some have no symptoms (asymptomatic), that ultimately usually endosalpingiosis is a coincidence condition found. The symptoms complained also depend on the location of the lesion; generally, complained symptoms are chronic pelvic pain, infertility, irregular menstruation, dyspareunia, gross hematuria, gastrointestinal tract obstruction, and jaundice. Endosalpingiosis could be symptomatic due to mechanical irritation of abdominal organs, for example in the case of large cysts of endosalpingiosis causes chronic pain in the waist, where the surgical removal of the cyst can relieve symptoms, such as cases which have been reported by Andreas $\mathrm{H}$ Scheel from Germany in 2013. [2, 6, 12]

In this case, the patient complained of lower abdominal pain caused by cystic mass of right paratubal growing larger and experiencing friction with other abdominal organs and the presence of rectum with uterine adhesions that cause the sensation of pain. Carried out adhesiolysis (release of adhesions) in order to reduce or eliminate the pain of his stomach. This patient also experienced secondary infertility, that after ten years of childbirth until now had never been pregnant again. The cause of infertility was most likely caused by a big right paratubal cyst causing pressure in the fallopian tube channel, which in turn causes a narrowing of the lumen of the tube, that the ovum transportation and sperm transport were disrupted.
Therapy in endosalpingiosis is basically not necessary because it is generally asymptomatic and has never been reported to undergo a transformation into a malignancy, although such therapy is performed depending on the condition and discovered cases such as ovarian cysts, brown cysts, fertility, pelvic pain, adhesions and dyspareunia. [6, 11] In this case we did bilateral cystectomy to improve reproductive function and eliminate the cause of the obstruction in her right fallopian tubes. We also carried out chromo tubal patency test and the results both tubes were patent.

Characteristics of endosalpingiosis are still difficult to diagnose and distinguished from ovarian malignancies as well as benign changes in the other ovary, that it is necessary to obtain a confirmation of histopathologic examination prior to the surgical procedure more aggressive. It is also necessary for clinicians to be more professional to avoid mistakes in diagnose and prevent over-treatment.

Routine follow-up in the case of endosalpingiosis is not necessary as long as there is no significant complaint from the patient, considering the case of endosalpingiosis has never been reported to undergo a transformation into a malignancy and there has been no written literature case of recurrence. However, in this patient, we followed up 3 months and the first 6 months post-surgery and abdominal pain was not felt anymore. Lack of knowledge and experience and prudence will be a natural progression of the lesion of Mullerian is a challenge for clinicians to conduct further research in the case of endosalpingiosis.

\section{Conclusion}

Endosalpingiosis is a rare case. The attention to this rare case will prevent us from making a wrong diagnosis and over-treatment.

\section{References}

[1] Rosa Bermejo, et al. Peritoneal Mullerian Tumor-Like (Endosalpingiosis-Leiomyomatosis Peritoneal): A Hardly Known Entity. Case Report in Obstertrics and Gynecology. 2012, volume 2012, P.1-3.

[2] Isabel Mesquita, et al. Endosalpingiosis of Choledochal duct. Journal Surgery. 2007.

[3] Katharine M Esselen, et al. Endosalpingiosis as it Relates to Tubal, Ovarian and Serous Neoplastic Tissues: An Immunohistochemical Study of Tubal and Mullerian Antigens. Gynecologic Oncology. 2013. vol.132, p.316-321.

[4] Zapardiel, et al. Endosalpingiosis Mimicking Recurrent Ovarian Carcinoma. Taiwanese Journal of Obstetrics \& Gynecology. 2012. Vol.51, p.660-662.

[5] Mohiedean Ghofrani, M. D. Ovary-nontumor Non-neoplastic cysts/ Other Endosalpingiosis. PathologyOutlines.com. 2014.

[6] Andreas H Scheel. Cystic endosalpingiosis Presenting as Chronic back Pain, a Case Report. Diagnostic Pathology.2013.

[7] Louise J. Magill,et al. Endocervicosis and Endosalpingiosis of The Urinary Bladder: A Case Report. British Journal of Medical \& Surgical Urology. 2011, p.128-130. 
[8] Seyran Yigit, et al. Tumor-Like Cystic Endosalpingiosis in The Myometrium: A Case Report and A Review of The Literature. Turkish Journal of Pathology. 2014. Vol.30 no.2, p.145-148.

[9] Prentice L, et al. What is Endosalpingiosis? American Society for Reproductive Medicine. 2012. p.942-947.

[10] Brigid Holloran-schwartz. Surgical Evaluation and Treatment of The Patient with Chronic Pelvic Pain. Obstet Gynecol Clin N. Am. 2014, p. 1-9.
[11] Wikipedia. Endosalpingiosis. 2014; p.1-3.

[12] Sangeeta Taneja, et al. MRI Appearance of Florid Cystic Endosalpingiosis of The Uterus: A Case Report. Korean Journal of Radiology. 2010. Vol.4, p.476-479. 